\title{
RBniCS - reduced order modelling in FEniCS
}

\author{
F. Ballarin, A. Sartori, G. Rozza
}

SISSA mathLab, International School for Advanced Studies, Trieste, Italy

SISSA

mathLab

Abstract
RBniCS [1] is a python-based library, man readable) code used for the au- terface. Indeed, it is a companion of
developed on top of FEniCS [3], aimed tomated solution of partial differential the introductory reduced basis hand-
at the developement of reduced order equations. Thanks to the features of book [2], and has been already used
models in the FEniCS environment. FEniCS the final user needs to prepare in doctoral classes within the "Mathe-
In particular, reduced order tech- a short code to carry out a reduced matical Analysis, Modelling, and Ap-
niques such as the certified reduced order simulation.
basis method and proper or- It is ideally suited for novice users well as for courses within the "Mas-
thogonal decomposition-Galerkin willing to learn reduced basis methods ter in High Performance Computing"
methods are implemented. The and reduced order modelling, thanks jointly offered by SISSA and Interna-
FEniCS project allows RBniCS to take to an object-oriented approach and tional Centre for Theoretical Physics
advantage of the high-level (e.g., hu- an intuitive and versatile python in- (ICTP).

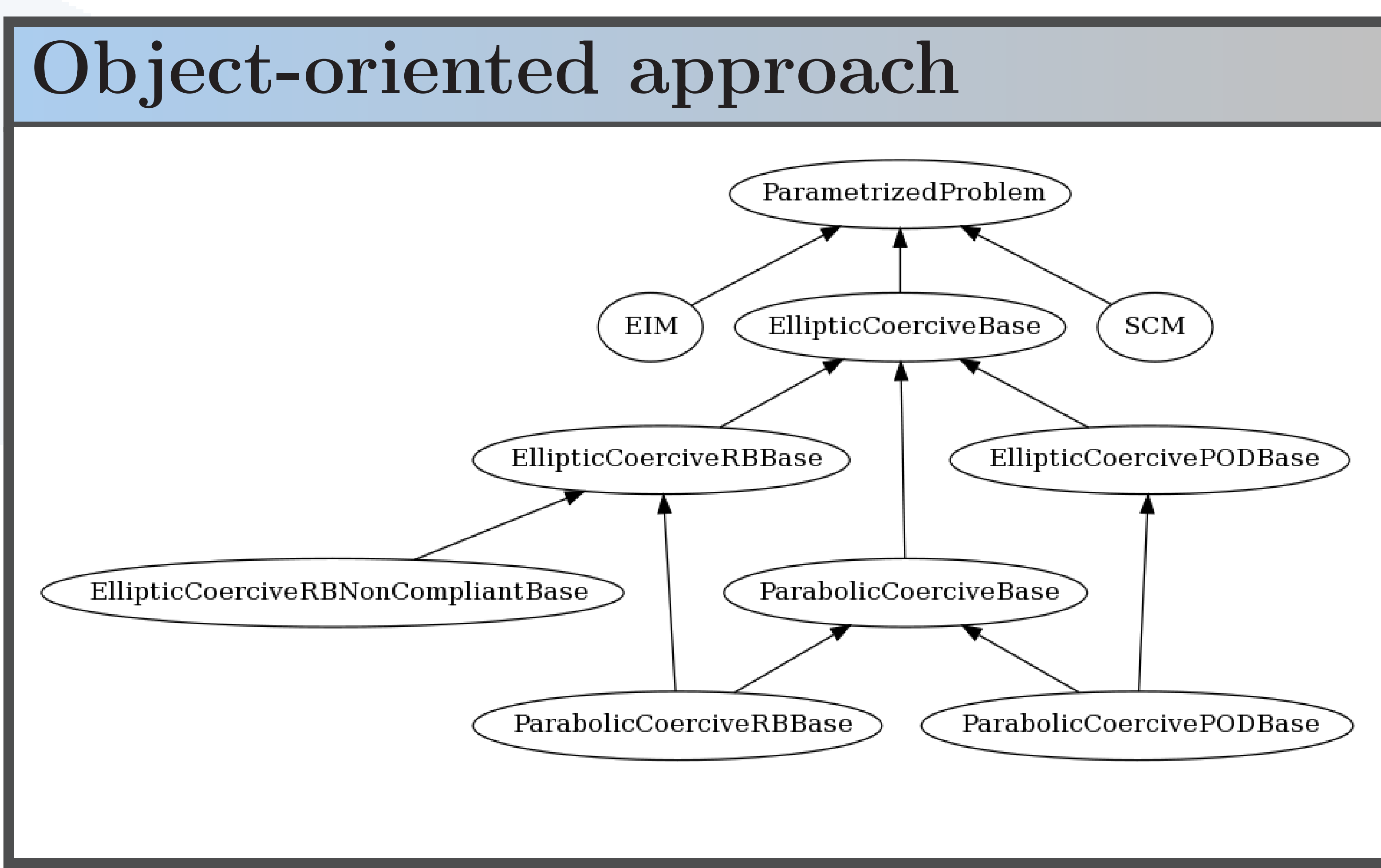

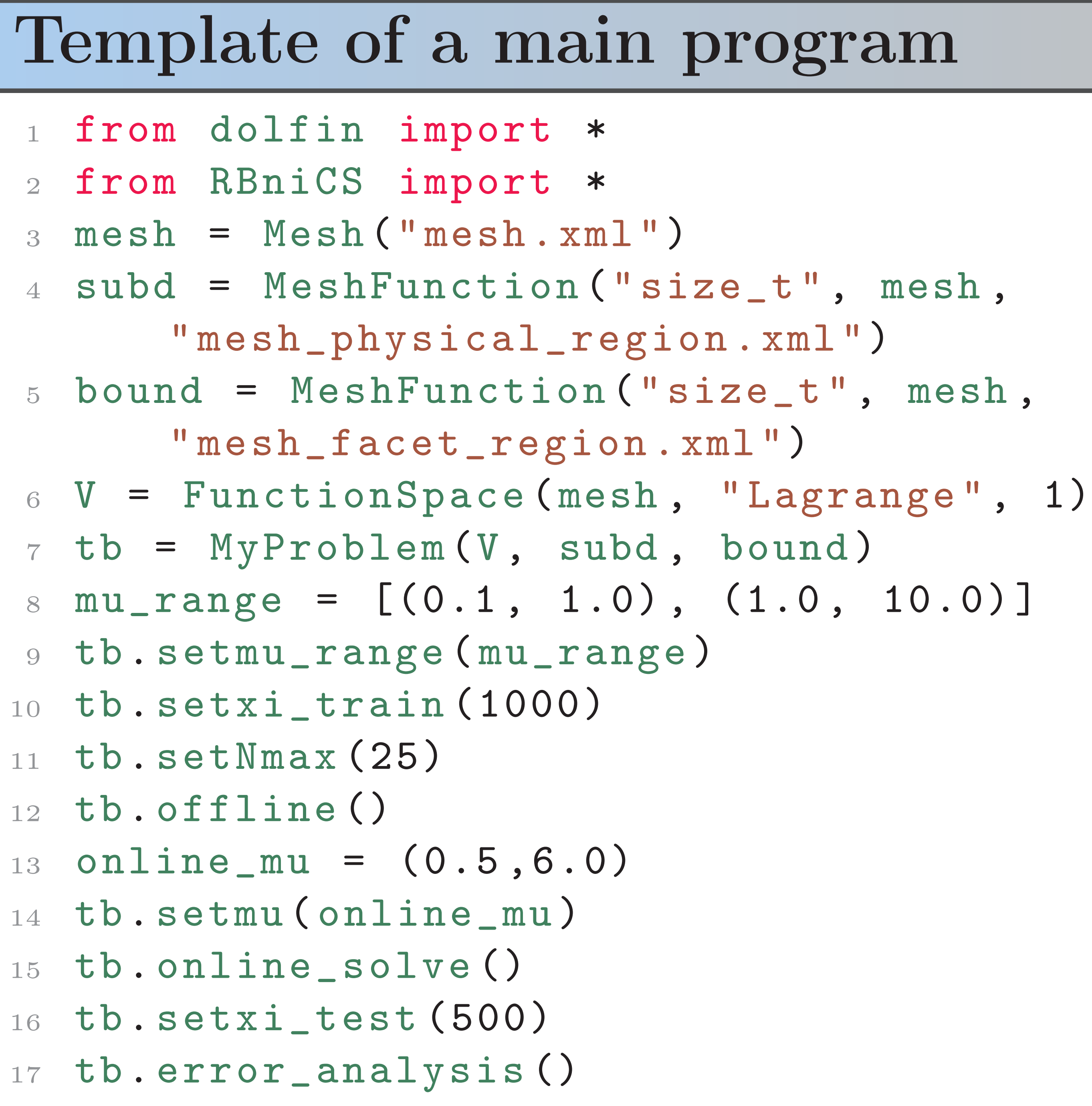

Template of a main program

\section{Tutorials 1 and 2: basics}

def _-_init__(self, V, subd, bound)

\# constructor

compute theta a (self):

$\operatorname{mu} 1=\operatorname{self} \cdot \operatorname{mu}[0]$

return (mu1, 1.)

def compute_theta_f(self):

$\operatorname{mu} 2=\operatorname{self} \cdot \operatorname{mu}[1]$

return (mu2, )

def assemble_truth-a (self)

$u=$ self.u

$\mathrm{a} 0=\operatorname{inner}(\operatorname{grad}(\mathrm{u}), \operatorname{grad}(\mathrm{v})) * d \mathrm{dx}(1)$

$A 0=\operatorname{assemble}(\mathrm{a} 0)$

a1 $=\operatorname{inner}(\operatorname{grad}(u), \operatorname{grad}(v)) * d x(2)$

$A 1=\operatorname{assemble}(a 1)$

\# Return

return ( $A 0, A 1)$

def assemble truth $f(\operatorname{self})$ :

$=\operatorname{self} \cdot v ; d s=\operatorname{self} \cdot d s$

\#Assemble

$\mathrm{f} 0=\mathrm{v} * \mathrm{ds}(1) ; \mathrm{F} 0=\operatorname{assemble}(f 0)$

\# Return

return (FO,

def get_alpha_lb(self)

return min(self.compute_theta_a())

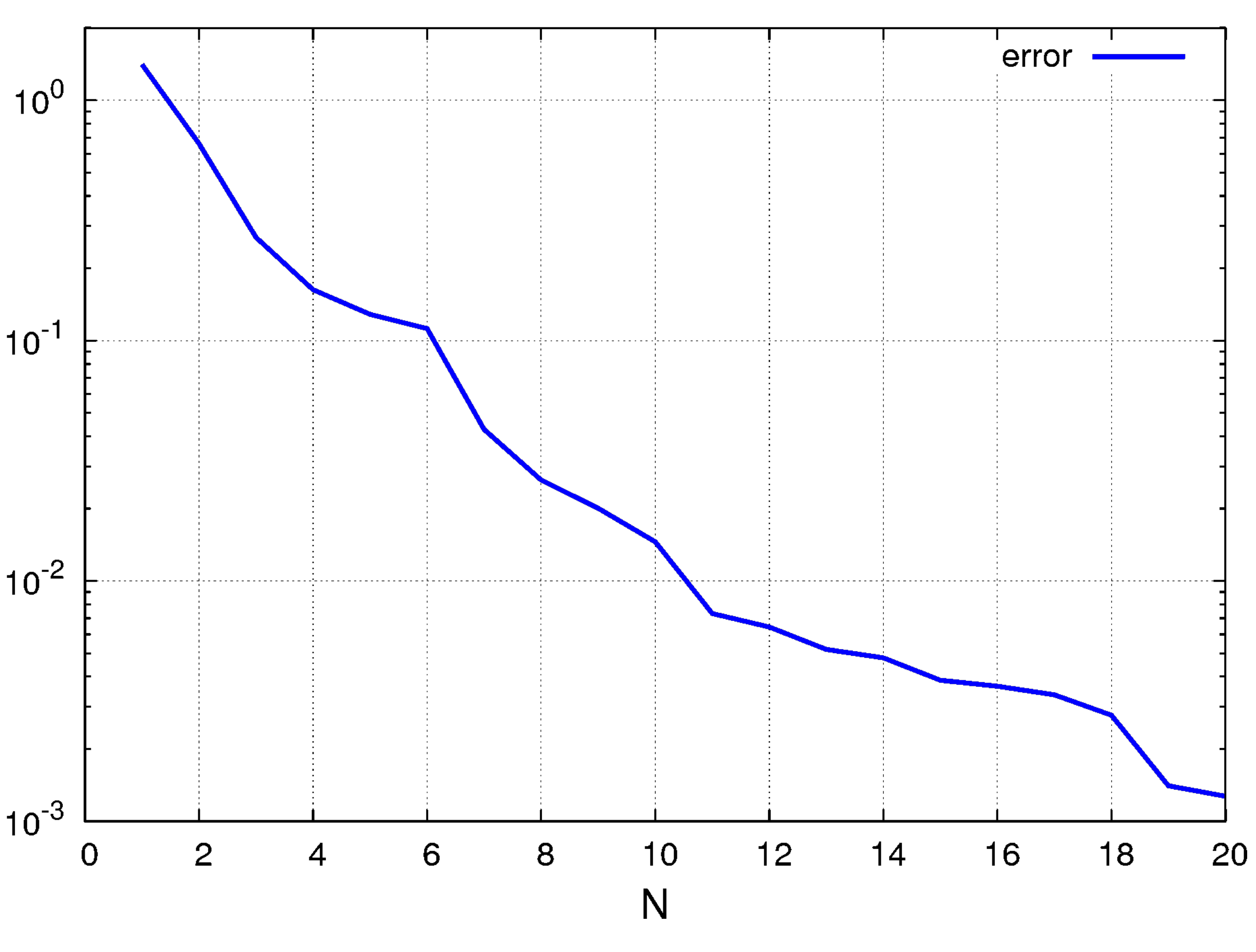

\section{How to get RBniCS}

RBniCS is freely available under the GNU LGPL, ver-

\section{aifta}

14:http://mathlab.sissa.it/rbnics
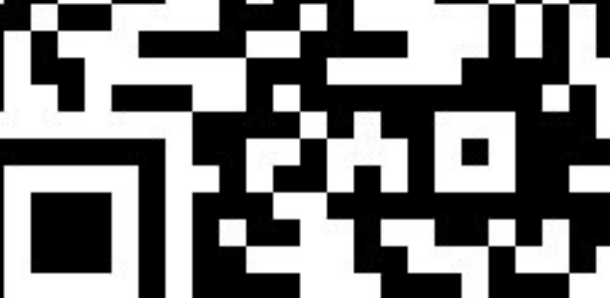

Tutorials 3 and 4: geometrical parametrization
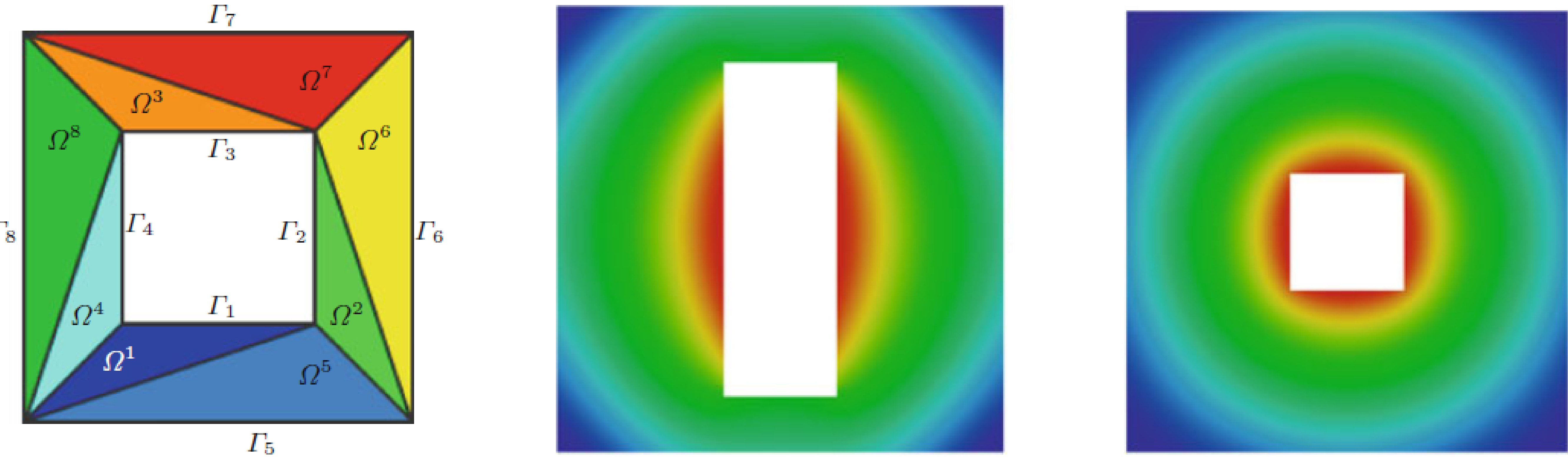

Tutorial 4: stability factor by SCM

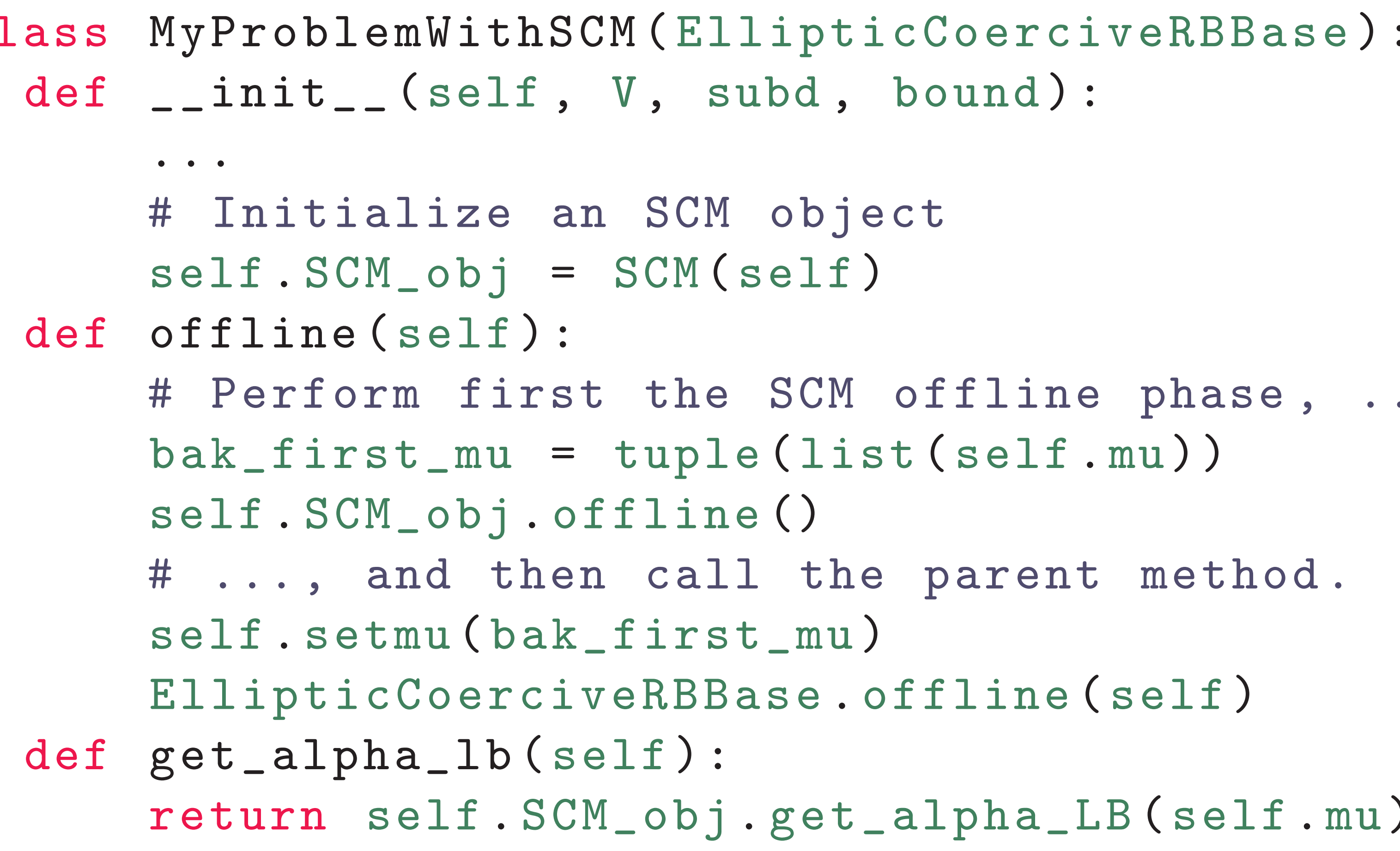

ass MyProblemWithSCM(EllipticCoerciveRBBase) :

def __init__(self, V, subd, bound):

\# Initialize an SCM object

self.SCM_obj = SCM (self)

def offline(self):

\# Perform first the SCM offline phase,

$b_{\text {bak_first_mu }}=t_{u p l e}\left(\right.$ list $\left.\left._{(\text {self }} \cdot \mathrm{mu}\right)\right)$

self.SCM_obj.offline()

and then call the parent method.

self. setmu (bak_first_mu)

EllipticCoerciveRBBase. offline(self)

def get_alpha_lb(self):

return self.SCM_obj.get_alpha_LB (self.mu)
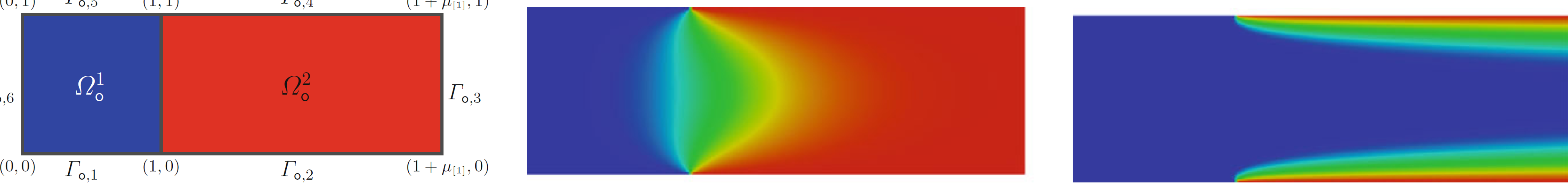

Tutorial 5: affinity assumption recovery by EIM
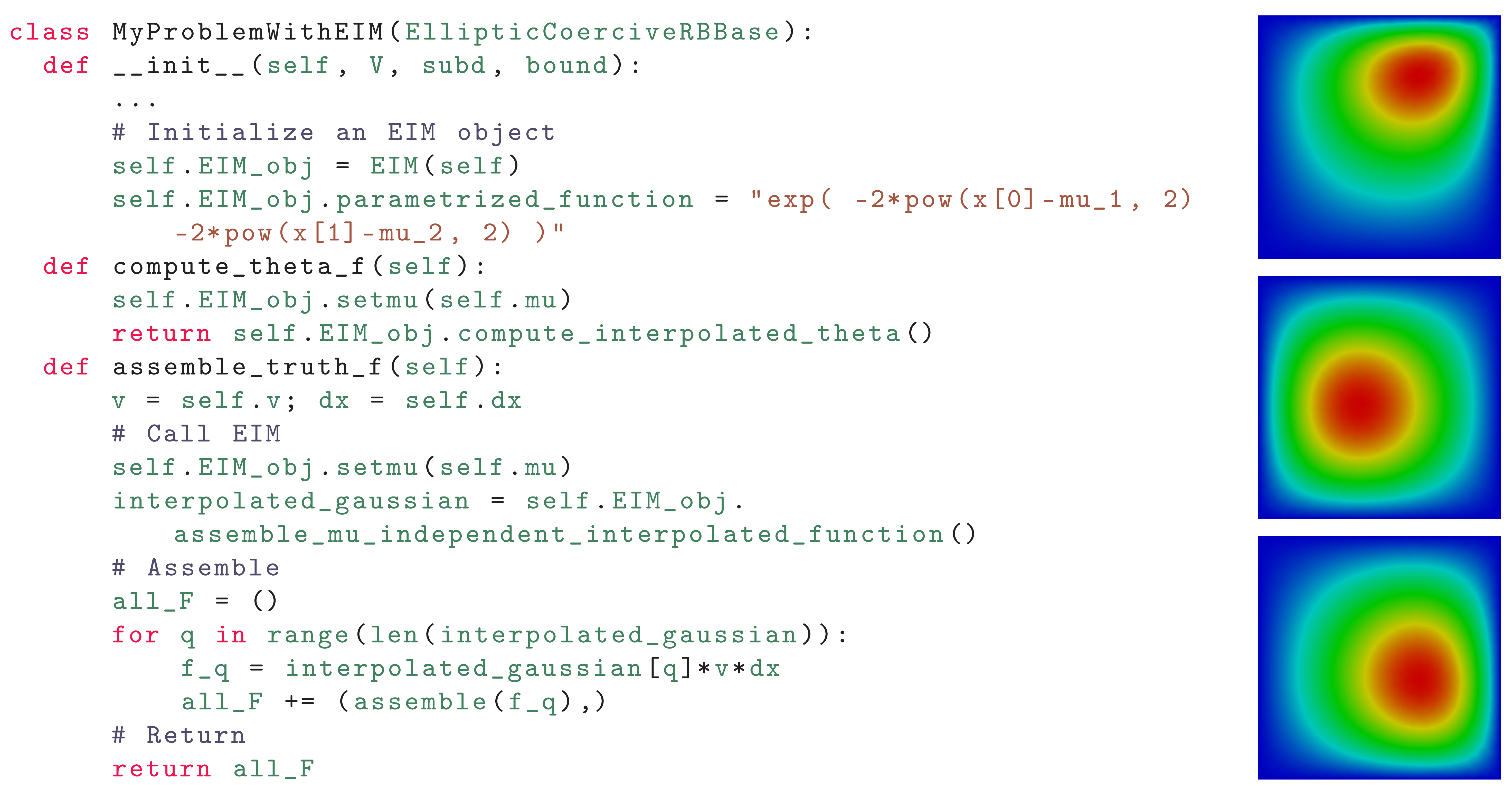

\section{References}

1] F. Ballarin, A. Sartori, and G. Rozza. RBniCS - reduced order modelling in FEniCS. http://mathlab.sissa.it/rbnics, 2015.

2] J. S. Hesthaven, G. Rozza, and B. Stamm. Certified Reduced Basis Methods for Parametrized Partial Differential Equations. SpringerBriefs in Mathematics. Springer, 2015

[3] A. Logg, K.-A. Mardal, G. N. Wells, et al. Automated Solution of Differential Equations by the Finite Element Method. Springer, 2012. 